Draft version November 6, 2018

Preprint typeset using $\mathrm{L}^{A} \mathrm{~T}_{\mathrm{E}} \mathrm{X}$ style emulateapj v. 04/17/13

\title{
THE POLLUTED ATMOSPHERE OF THE WHITE DWARF NLTT 25792 AND THE DIVERSITY OF CIRCUMSTELLAR ENVIRONMENTS *
}

\author{
S. Vennes And A. KAWKa \\ Astronomický ústav, Akademie věd České republiky, Fričova 298, CZ-251 65 Ondřejov, Czech Republic \\ Draft version November 6, 2018
}

\begin{abstract}
We present an analysis of X-Shooter spectra of the polluted, hydrogen-rich white dwarf NLTT 25792. The spectra show strong lines of calcium (Ca H\&K, near-infrared calcium triplet, and Ca I $\lambda 4226$ ) and numerous lines of iron along with magnesium and aluminum lines from which we draw the abundance pattern. Moreover, the photospheric Ca H\&K lines are possibly blended with a circumstellar component shifted by $-20 \mathrm{~km} \mathrm{~s}^{-1}$ relative to the photosphere. A comparison with a sample of four white dwarfs with similar parameters show considerable variations in their abundance patterns, particularly in the calcium to magnesium abundance ratio that varies by a factor of five within this sample. The observed variations, even after accounting for diffusion effects, imply similar variations in the putative accretion source. Also, we find that silicon and sodium are significantly underabundant in the atmosphere of NLTT 25792, a fact that may offer some clues on the nature of the accretion source.

Keywords: stars: abundances — stars: atmospheres — stars: individual (NLTT 25792) — white dwarfs
\end{abstract}

\section{INTRODUCTION}

The presence of heavy elements in the atmosphere of many cool white dwarfs is attributed to circumstellar debris that are continuously or intermittently accreted onto the white dwarf surface (see Zuckerman et al. 2003). Questions remain as to the phase and composition of the accreted material and its effect on the white dwarf abundance pattern. Zuckerman et al. (2003) and Koester et al. (2005) estimate that approximately $25 \%$ of cool, H-rich white dwarfs show the presence of metal lines in their spectra, while Zuckerman et al. (2010) found that a similar fraction of He-rich white dwarfs show metal lines. Kilic et al. (2006) explored the link between heavy element pollution in white dwarfs and the presence of a warm, near-infrared (IR) debris disk feeding the photosphere as in the case of the proto-typical dusty, polluted white dwarf G29-38 (Jura 2003). However, Farihi et al. (2009) reported that only $21 \%$ of polluted white dwarfs have mid-IR excess consistent with a circumstellar disk and they also noted an increased likelihood of a midIR excess for objects with a higher calcium abundance $(\log \mathrm{Ca} / \mathrm{H}(\mathrm{e}) \gtrsim-8.0)$. Actually, Farihi et al. (2010) observed several polluted H-rich (DAZ) and He-rich (DZ) white dwarfs with Spitzer and found that the debris disks have varying thickness and, therefore, some of these may be narrow enough to escape detection. Moreover, Jura (2008) shows that polluted white dwarfs that do not exhibit an IR excess may still be experiencing gas accretion that originated from the tidal destruction of a large number of small asteroids. Indeed, gaseous disks around white dwarfs were detected via near-IR calcium triplet emission (Gänsicke et al. 2006, 2007).

Various accretion scenarios or many types of sources may be involved. For example, the immediate environment of white dwarf stars is likely composed of remnant

\footnotetext{
* Based on observations collected at the European Organisation for Astronomical Research in the Southern Hemisphere, Chile under programme ID 091.D-0267.
}

bodies that survived post-asymptotic giant branch evolution. These bodies may be low-mass stellar (see, e.g., Kawka et al. 2008) or sub-stellar companions (see, e.g., Steele et al. 2013), or disrupted planetary systems, i.e., objects that are otherwise quite common. The formation of a debris disk could mix material and produce an abundance pattern averaged over several constituents. Consequently, the observed abundance would be representative of the stellar neighborhood. However, single body accretion could deliver a greater diversity of abundance patterns (see, e.g., Zuckerman et al. 2011).

The accretion history and diffusion time-scales mingle in a complex manner (Dupuis et al. 1992). Koester (2009) considered three possible sequences of events: One of continuous accretion and built-up toward diffusive equilibrium, one at diffusive equilibrium, and, after extinction of the accretion source, one of decline in photospheric abundances. Therefore, the observed abundance pattern, i.e., the photospheric abundance ratios, may differ considerably from the source pattern, and a case-by-case study of DAZ white dwarfs may yet reveal considerable abundance variations.

On last count (see, e.g., Zuckerman et al. 2003; Koester et al. 2005), cool DAZ white dwarfs $\left(T_{\text {eff }} \lesssim 8000\right.$ $\mathrm{K}$ ) are outnumbered at least 1:3 by the DZ white dwarfs (see, e.g., Dufour et al. 2007). Interestingly, the number of identified DAZ white dwarfs in the local sample of Giammichele et al. (2012) is comparable to that of DZ white dwarfs (11 versus 13 stars), although the DAZ count corresponds to a much smaller fraction of all H-rich white dwarfs than that of their He-rich counterparts, i.e., one tenth versus one third, respectively. Clearly, many polluted $\mathrm{H}$-rich white dwarfs remain to be recognized as such in the local sample. Koester (2009) cites difficulties in detecting weak metal lines in the opaque, neutral hydrogen environment of cool DA white dwarfs. However, recent spectroscopic surveys of local white dwarf candidates (see, e.g., Kawka \& Vennes 2006, 2012a) have uncovered several new cool DAZ white 
Table 1

Photometry

\begin{tabular}{|c|c|c|}
\hline Survey and band ${ }^{\mathrm{a}}$ & $\lambda$ effective & $m$ \\
\hline$G A L E X \mathrm{NUV}$ & $2271 \AA$ & $17.78 \pm 0.03$ \\
\hline SDSS $u$ & $3551 \AA$ & $16.540 \pm 0.007$ \\
\hline SDSS $g$ & $4686 \AA$ & $16.079 \pm 0.004$ \\
\hline SDSS $r$ & $6116 \AA$ & $15.974 \pm 0.004$ \\
\hline $\operatorname{SDSS} i$ & $7481 \AA$ & $15.984 \pm 0.005$ \\
\hline SDSS $z$ & $8931 \AA$ & $16.062 \pm 0.007$ \\
\hline 2MASS $J$ & $1.235 \mu \mathrm{m}$ & $15.521 \pm 0.050$ \\
\hline 2MASS $H$ & $1.662 \mu \mathrm{m}$ & $15.401 \pm 0.111$ \\
\hline 2MASS $K$ & $2.159 \mu \mathrm{m}$ & $15.943 \pm 0.255$ \\
\hline$W I S E W 1$ & $3.353 \mu \mathrm{m}$ & $15.268 \pm 0.046$ \\
\hline$W I S E W 2$ & $4.603 \mu \mathrm{m}$ & $15.051 \pm 0.103$ \\
\hline \multicolumn{3}{|c|}{$\begin{array}{l}\text { a GLEX GR6/GR7 photometry } \\
\text { galex.stsci.edu/GalexView/; SDSS } \\
\text { Catalog, Release } 7 \text { (Abazajian et al. } 2009) ; 2 \text { MASS } \\
\text { photometry (Skrutskie et al. 2006); (WISE) pho- } \\
\text { tometry (Cutri et al.|2012). }\end{array}$} \\
\hline
\end{tabular}

dwarfs (Kawka \& Vennes 2011, 2012b).

In this context, we present an analysis of the polluted, otherwise hydrogen-rich white dwarf NLTT 25792 from the revised New Luyten Two-Tenth catalog (Salim \& Gould 2003). Kawka et al. (2011) and Gianninas et al. (2011) reported the detection of a strong $\mathrm{Ca} \mathrm{K}$ line but without other identifiable elements. This object is also known in the Edinburgh-Cape catalog as EC 10542-2236 (Kilkenny et al. 1997) and in the Luyten-Palomar catalog as LP 849-31. NLTT 25792 is located above the Galactic plane $(l=271.1687, b=$ $+32.8146)$ and toward a relatively tenuous line of sight in the interstellar medium $\left(E_{B-V}=0.0595\right.$, Schlegel et al. 1998). The proper motion quoted in the rNLTT cata$\log$ is $\left(\mu_{\alpha} \cos \delta, \mu_{\delta}\right)=(-72,293)$ mas $\mathrm{yr}^{-1}$. Section 2 details our recent observations of this object using the X-shooter spectrograph at the European Southern Observatory, and in Section 3 we present an analysis of this and other related polluted white dwarfs. We conclude in Section 4.

\section{OBSERVATIONS}

We obtained two consecutive sets of echelle spectra using the X-shooter spectrograph (Vernet et al. 2011) attached to the UT2 (Kueyen) at Paranal Observatory on UT 2013 May 10. The observations were conducted in clear sky conditions at an average airmass of 1.24 in the first set and 1.60 in the second. The seeing conditions were $1.20 \operatorname{arcsec}$ on average $(\sigma=0.11$ arcsec $)$ during the first observation and $1.36 \operatorname{arcsec}(\sigma=0.13$ arcsec) during the second. The spectra were obtained at the parallactic angle and in the "stare" mode, with the slit width set to $0.5,0.9$ and 0.6 arcsec for the UVB, VIS and NIR arms, respectively. This arrangement delivered a resolution of $R=\lambda / \Delta \lambda=9100,8800$ and 6200 for the UVB, VIS and NIR arms, respectively, for nominal wavelength ranges

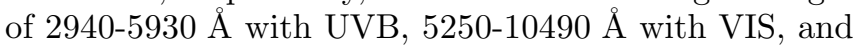
$0.98-2.48 \mu \mathrm{m}$ with NIR. For each set, the exposure times were 2940 and $3000 \mathrm{~s}$ for the UVB and VIS arms, respectively. For the NIR arm we obtained five exposures of $600 \mathrm{~s}$ each. An analysis of the NIR observations will be presented elsewhere.

We reduced the observations using the $\mathrm{X}$-shooter reduction pipeline under the ESO Recipe Flexible Exe- cution Workbench (Reflex). Details of the X-shooter pipeline and Reflex are available in the ESO documents VLT-MAN-ESO-14650-4840 (issue 12.0) and VLT-MANESO-19540-5899 (issue 2.0). The extracted spectra were resampled with $0.2 \AA$ bins, corresponding to half of a resolution element in the UVB spectra, and one third of a resolution element in the VIS spectra. The signal-tonoise ratio (SNR) achieved in the co-added UVB spectrum at $\lambda=3100 \AA$ was $\mathrm{SNR} \sim 10$ per bin thereby setting the lowest usable wavelength. The measured SNR reached $\sim 30$ per bin at $3500 \AA, \sim 80$ at 3900 and 4200 $\AA$, and $\sim 90$ at $5000 \AA$. The measured SNR reached $\sim 54$ per bin in the co-added VIS spectrum at $5900 \AA, \sim 70$ at $6600 \AA$, and $\sim 90$ at $8600 \AA$.

Table 1 lists available photometric measurements from the Galaxy Evolution Explorer (GALEX) sky survey, the Sloan Digital Sky Survey (SDSS), the Two Micron All Sky Survey (2MASS), and the Wide-field Infrared Survey Explorer (WISE).

\subsection{Comparison Sample}

Also, we obtained a series of HIRES spectra ( $R=25000$ to 40000$)$ for a set of closely related DAZ white dwarfs: WD 0208+396 (G74-7), WD 0354+463, WD $1257+278$, and WD $1455+298$ (Zuckerman et al. 2003, 2011). Zuckerman et al. (2003) published the spectra for WD 0208+396 (with the Keck Observatory Archive, KOA, label HI.19990813.45167), WD 0354+463 (HI.19980123.32497), and WD 1455+298 (HI.19980624.32907). We supplemented the published data set with spectra obtained from the KOA for WD $1257+278$ (HI.20100327.33502, HI.20100327.35353, and HI.20100328.32028) and WD 1455+298 (HI.20060617.24712, HI.20060617.27218, HI.20060617.29698, and HI.20060617.32157). We used the weighted average of the spectra in our abundance analysis.

A comparative abundance analysis should help us identify common properties of the sample, or, alternatively, features that are peculiar to NLTT 25792. The spectral energy distributions (SED) of the comparison stars (see Appendix 1) are well reproduced by a single star model or in the case of WD $0354+463$ by a binary star template. The SED of WD $1455+298$ shows a weak IR excess in the WISE bands $(\lambda \gtrsim 3 \mu \mathrm{m})$ suggesting the presence of warm dust; Farihi et al. (2008) noted a weak excess in Spitzer IRAC measurements at $\lambda \gtrsim 5 \mu \mathrm{m}$ and inferred a dust temperature of $400 \mathrm{~K}$.

The prototypical DAZ white dwarf G74-7 (WD 0208+396 Lacombe et al. 1983; Billeres et al. 1997; Zuckerman et al. 2003) lies in the Galactic plane $(l=139.2, b=-20.4)$ at a distance of $\sim 17 \mathrm{pc}$ $(\pi=0.0598 \pm 0.0035$ van Altena et al. 1995) $)$ and toward a relatively tenuous line-of-sight $\left(E_{\mathrm{B}-\mathrm{V}}=0.0485\right)$.

The DAZ white dwarf WD 0354+463 (Zuckerman et al. 2003) and its dM7 companion form an unresolved, possibly close pair (sep. $<0.8$ AU, Farihi et al. 2006). Zuckerman et al. (2003) noted emission in the $\mathrm{H} \beta$ line core, but no radial velocity variations have been reported to date. The presence of heavy elements in the atmosphere of the white dwarf may be attributed to a wind-accretion mechanism (Zuckerman et al. 2003; Debes 2006). The binary 
lies in the Galactic plane $(l=153.2, b=-5.1)$ and in a relatively dense line-of-sight $\left(E_{\mathrm{B}-\mathrm{V}}=0.642\right)$. The SED of WD $0354+463$ is well reproduced by the combination of a DAZ white dwarf model and a M7 template constructed using optical/IR spectroscopy of VB 8 (=GJ 644 C; Tinney \& Reid 1998; Rayner et al. 2009; Cushing et al. 2005). The star VB 8 is located at a distance of $6.5 \pm 0.1 \mathrm{pc}$ (Thorstensen 2003). This exercise demonstrates that the optical/ultraviolet part of the spectrum is dominated by the DAZ white dwarf. Therefore, the Balmer line analysis is probably correct although weak emission line cores should be excluded from the analysis. However, the spectral decomposition based on the M7 template implies a larger distance $(d=43.2 \pm 0.7 \mathrm{pc})$ than estimated using the white dwarf absolute magnitude $(d=33.4 \pm 2.5 \mathrm{pc})$. The distance estimates are reconciled by adopting for the companion an absolute magnitude $M_{K}=10.32$, or a sub-type between M8 and M9 (Kirkpatrick \& McCarthy 1994) and $0.56 \mathrm{mag}$ fainter than the template itself $\left(M_{K}(\mathrm{VB})=9.78 \mathrm{mag}\right.$, Kirkpatrick \& McCarthy 1994). Alternatively, the white dwarf itself could be 0.56 mag brighter implying a lower surface gravity than measured spectroscopically.

Finally, both WD 1257+278 (Zuckerman et al. 2003, 2011) and WD 1455+298 (Zuckerman et al. 2003) are relatively more distant at $\sim 35 \mathrm{pc}$ (van Altena et al. 1995), but at high Galactic latitudes of $b=88.1$ and 62.1, respectively, and correspondingly low extinction in the line-of-sight ( $E_{B-V}=0.0095$ and 0.0177 , respectively). No IR calcium triplet emission have been detected in the HIRES spectra of WD $1257+278$ and WD $1455+298$.

\section{ANALYSIS}

We based our analysis of the Balmer and heavy element line profiles on a grid of model atmospheres in local thermodynamic equilibrium that include convective energy transport. We employed the mixing-length formalism with parameters ML2 and $\alpha=0.6$. Details of Balmer line profiles are provided by Kawka \& Vennes (2006). Heavy-element contributions to the electron density are included with the metallicity varying from $[\mathrm{X} / \mathrm{H}] \equiv \log \mathrm{X} / \mathrm{H}-\log \mathrm{X} / \mathrm{H}_{\odot}=-4.0$ to -2.0 , where $\mathrm{X}$ includes the 18 most abundant species from carbon to zinc. Based on the model atmosphere structure we computed detailed heavy element line profiles using Voigt functions and state-of-the-art oscillator strengths and broadening parameters (see details in Kawka \& Vennes 2012b); In most cases, collisions with neutral hydrogen atoms, and, to a lesser extent, electrons dominate the line profiles.

The solar abundance scale employed in the present work was built using the compilations of Asplund et al. (2009) and Lodders et al. (2009). Lodders et al. (2009) provide a critical compilation of meteoritic, i.e., the CI carbonaceous chondrites, and solar photospheric abundances. Employing somewhat different criteria, Asplund et al. (2009) list solar photospheric abundances that differ on average by only 0.005 dex with a dispersion of 0.04 dex from those of Lodders et al. (2009) for a group of abundant elements comprising $\mathrm{Na}, \mathrm{Mg}, \mathrm{Al}, \mathrm{Si}$, $\mathrm{Ca}$, and Fe. Note that for that same group of elements, the CI-chondrites and solar photospheric abundances are nearly identical with differences no larger than 0.02 dex (Lodders et al. 2009). Therefore, in this work, we use the straight average of the solar photospheric abundances of Asplund et al. (2009) and the CI-chondrites and solar photospheric abundances of Lodders et al. (2009). We will refer to this joint scale as the "solar abundances": $\log \mathrm{Na} / \mathrm{H}_{\odot}=-5.72, \log \mathrm{Mg} / \mathrm{H}_{\odot}=-4.44$, $\log \mathrm{Al} / \mathrm{H}_{\odot}=-5.54, \log \mathrm{Si} / \mathrm{H}_{\odot}=-4.48, \log \mathrm{Ca} / \mathrm{H}_{\odot}=$ $-5.67, \log \mathrm{Fe} / \mathrm{H}_{\odot}=-4.53$. Finally, Lodders et al. (2009) proposes to scale proto-solar (or "solar system") abundances from solar abundances using the logarithmic relation $X_{0}=X+0.053$. Abundance ratios are not affected by this scaling and the proto-solar abundances will not be considered further.

We fitted the Balmer line profiles and extracted the effective temperature and surface gravity (Section 3.2) and constrained the abundance of individual elements (Section 3.3) using $\chi^{2}$ minimization techniques.

\subsection{NLTT 25792: Line Identifications and Radial Velocity}

Figure 1 shows segments of the UVB X-shooter spectrum. Notable features include the $\mathrm{Ca} H \& \mathrm{~K}$ doublet and the upper Balmer lines $(\mathrm{H} \gamma$ to $\mathrm{H} 8)$ and numerous Fe I lines. The average velocity of 49 spectral lines (Table 2) found between $\sim 3440$ and $\sim 8662 \AA$ is $24.7 \mathrm{~km} \mathrm{~s}^{-1}$ with a dispersion of only $3.6 \mathrm{~km} \mathrm{~s}^{-1}$. Estimating the gravitational redshift of the white dwarf at $31.7 \pm 1.5$ $\mathrm{km} \mathrm{s}^{-1}$ (Section 3.2), the radial velocity of the white dwarf is $v_{r}=-7.0 \pm 3.9 \mathrm{~km} \mathrm{~s}^{-1}$. The velocity difference between the two consecutive $(\Delta t \approx 1 \mathrm{hr})$ exposures is $v_{1}-v_{2}=-2.2 \mathrm{~km} \mathrm{~s}^{-1}$ with a dispersion of $4.1 \mathrm{~km} \mathrm{~s}^{-1}$.

Figure 2 compares the main spectral features in NLTT 25792 and the comparison stars. These objects share many important spectral features, most notably dominant $\mathrm{Ca} \mathrm{H} \& \mathrm{~K}$ doublets and rich iron line spectra. A shift of the Ca K line in NLTT 25792 relative to the other two stars is apparent when lining-up the spectra with other narrow metal lines. The IR calcium triplet in NLTT 25792 is in absorption with no evidence of an emission component.

\subsection{Atmospheric Parameters and Spectral Energy Distribution (SED) of NLTT 25792}

We fitted the Balmer line profiles, $\mathrm{H} \beta$ to $\mathrm{H}_{10}$ excluding $\mathrm{H} \epsilon$, in the X-shooter and FORS1 (Kawka et al. 2011) spectra independently. Our new measurements, $\left(T_{\text {eff }}, \log g\right)=(7900 \pm 20 \mathrm{~K}, 8.09 \pm 0.04)$ with Xshooter and $(7910 \pm 20 \mathrm{~K}, 7.96 \pm 0.05)$ with FORS1, are in excellent agreement with the measurements of Gianninas et al. (2011): $\quad\left(T_{\text {eff }}, \log g\right)=(7910 \pm$ $118 \mathrm{~K}, 8.05 \pm 0.08)$. We adopted the weighted averages of the measurements: $\left(T_{\text {eff }}, \log g\right)=(7903 \pm 16 \mathrm{~K}, 8.04 \pm$ $0.03)$. Based on these parameters, the mass of the white dwarf is $0.618 \pm 0.018 M_{\odot}$ and the radius is $0.0124 \pm$ $0.0002 R_{\odot}$ with a cooling age of $1.2 \mathrm{Gyr}$. The absolute magnitude in the SDSS $r$ band, $M_{r}=13.18 \pm 0.04 \mathrm{mag}$, locates the star at a distance of $d=36.2 \pm 0.7 \mathrm{pc}$.

The SED of NLTT 25792 (Fig. 3) is characteristic of a $7900 \mathrm{~K}$ white dwarf without a low-mass stellar companion. Also, the SED does not show a warm disk often encountered in the IR spectra of polluted white dwarfs (Kilic et al. 2006; Farihi et al. 2009). In fact, the measured IR flux shows an unexplained dip measured in the 


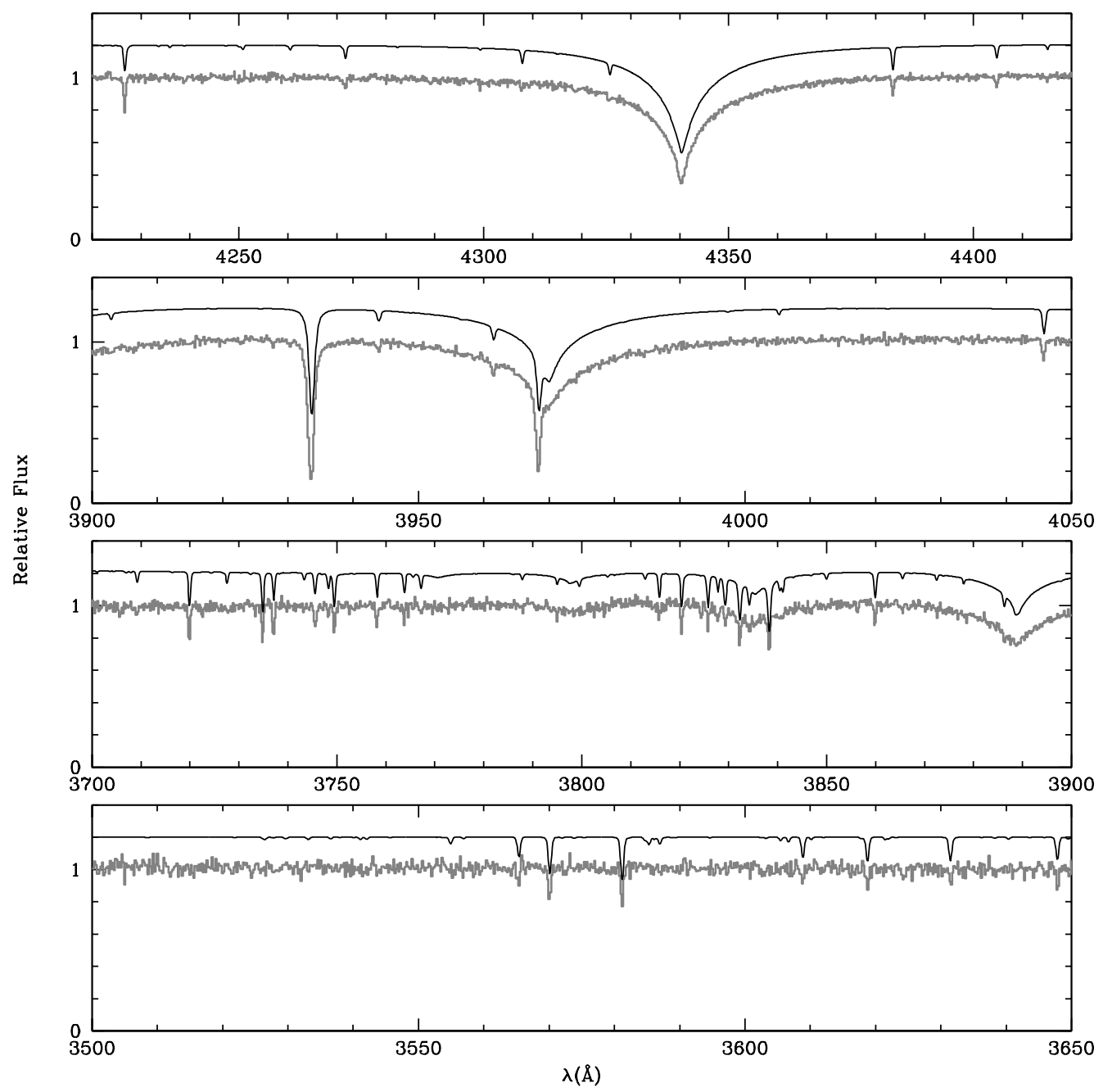

Figure 1. Sections of the X-shooter spectra from 3500 to $4420 \AA$ and showing many spectral features listed in Table 2 The data are compared to a representative model with $[\mathrm{X} / \mathrm{H}]=-2.5$.

K-band. However, we noted the possible effect of interstellar reddening on the ultraviolet part of the SED with $E_{B-V}=0.016$ using the models of Cardelli et al. (1989) and $R_{V}=3.1$.

\subsection{Heavy Element Abundance Pattern in NLTT 25792}

We measured the abundance of magnesium, aluminum, calcium and iron using spectral lines listed in Table 2 We fitted the line profiles rather than the equivalent widths. These are provided as indicative of the relative line strengths. Following a procedure adopted in the past (Kawka \& Vennes 2012b), we employed the broadening parameter $\Gamma$ from Barklem et al. (2000) added to Stark and natural broadening parameters. The resulting Voigt profiles are convolved with Gaussian profiles set to match the spectral resolution. The measured abundances are listed in Table 3. We noted a discrepancy between the calcium abundance based on the $\mathrm{Ca} \mathrm{K}$ line $([\mathrm{Ca} / \mathrm{H}]=-2.14 \pm 0.06)$ and that based on $\mathrm{Ca} \mathrm{I}$ and the Ca II triplet $([\mathrm{Ca} / \mathrm{H}]=-2.40 \pm 0.06)$. We excluded the $\mathrm{Ca} H \& \mathrm{~K}$ lines from the abundance measurement and further investigate the question in Section 3.5.

The upper limit to the equivalent width of Si I $\lambda 3905.523$ line is $\sim 10 \mathrm{~m} \AA$ and the upper limits to the $\mathrm{Na} I \lambda \lambda 5889.951,5895.924$ lines are $\sim 15 \mathrm{~mA}$. These estimates correspond to $3 \sigma$ upper limits, i.e., $3 \times \Delta \lambda / \mathrm{SNR}$, where $\Delta \lambda$ is the full width of a resolution element and the SNR is measured within bins the size of a resolution element. For example, $\Delta \lambda=0.50 \AA$ near Fe $\mathrm{I} \lambda 4415$ and the measured SNR is 90 per $0.2 \AA$ bin, or 142 per resolution element, corresponding to a minimum equivalent width of $\sim 10 \mathrm{~m} \AA$. The corresponding silicon and sodium abundance upper limits are $\log \mathrm{Si} / \mathrm{H} \lesssim-7.5$ $([\mathrm{Si} / \mathrm{H}] \lesssim-3.0)$ and $\log \mathrm{Na} / \mathrm{H} \lesssim-8.8([\mathrm{Na} / \mathrm{H}] \lesssim-3.1)$. Silicon and sodium are markedly depleted relative to all other elements ( $\mathrm{Mg}, \mathrm{Al}, \mathrm{Ca}$, and $\mathrm{Fe}$ ) on the solar abundance scale. Also, we noted a possible ISM component to the $\mathrm{Na} \mathrm{I} \lambda 5889.951$ line at $-13 \mathrm{~km} \mathrm{~s}^{-1}$ and with an equivalent width of $35 \mathrm{m \AA}$. We did not attempt to con- 

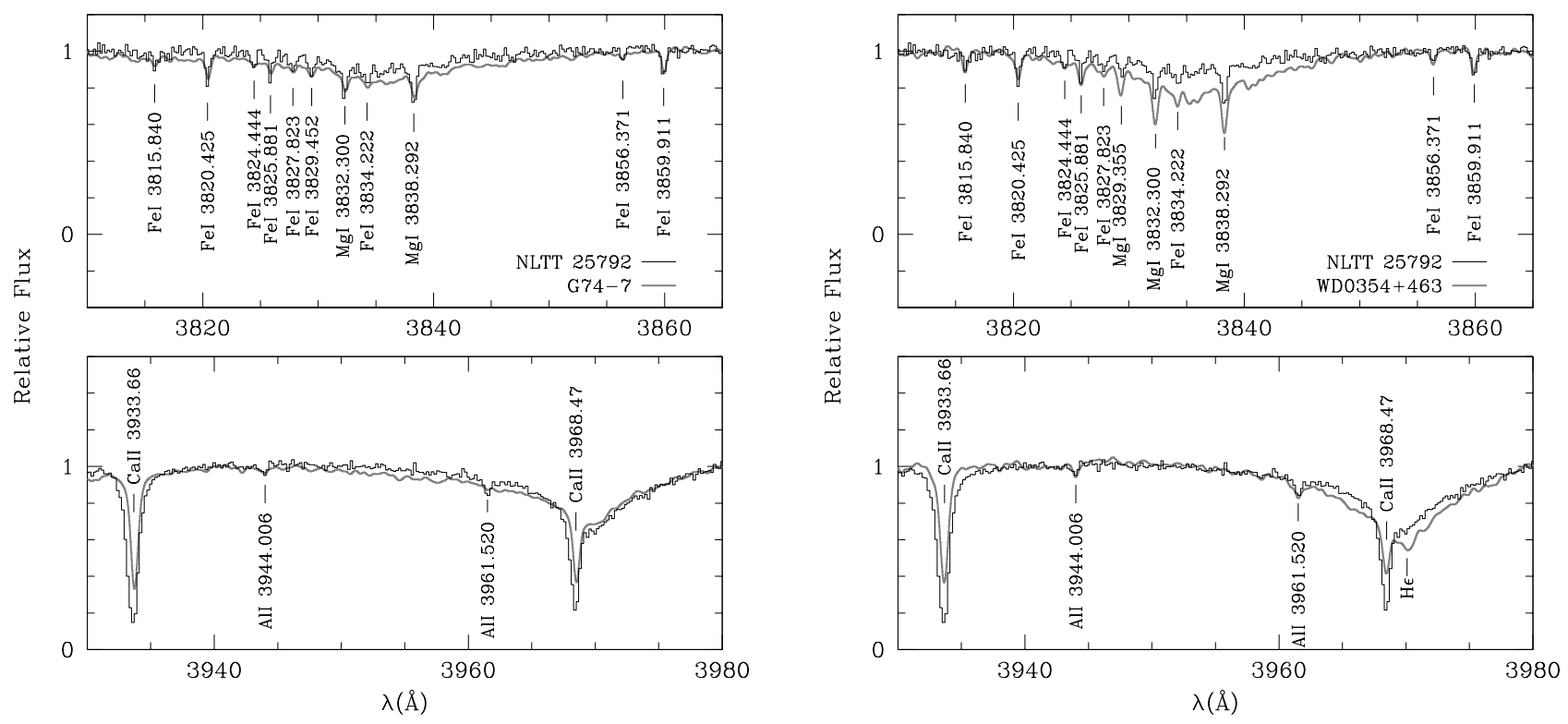

Figure 2. Comparing the Ca H\&K doublet (lower panel) and ultraviolet Mg I/Fe I lines (top panel) in the X-shooter spectra of NLTT 25792 (black lines) with Keck/HIRES spectra other DAZ white dwarfs (grey lines): (left panels) G74-7 (WD 0208+396) and (right panels) WD 0354+463 (DAZ+dM). The HIRES spectra have been degraded to the X-shooter resolution.

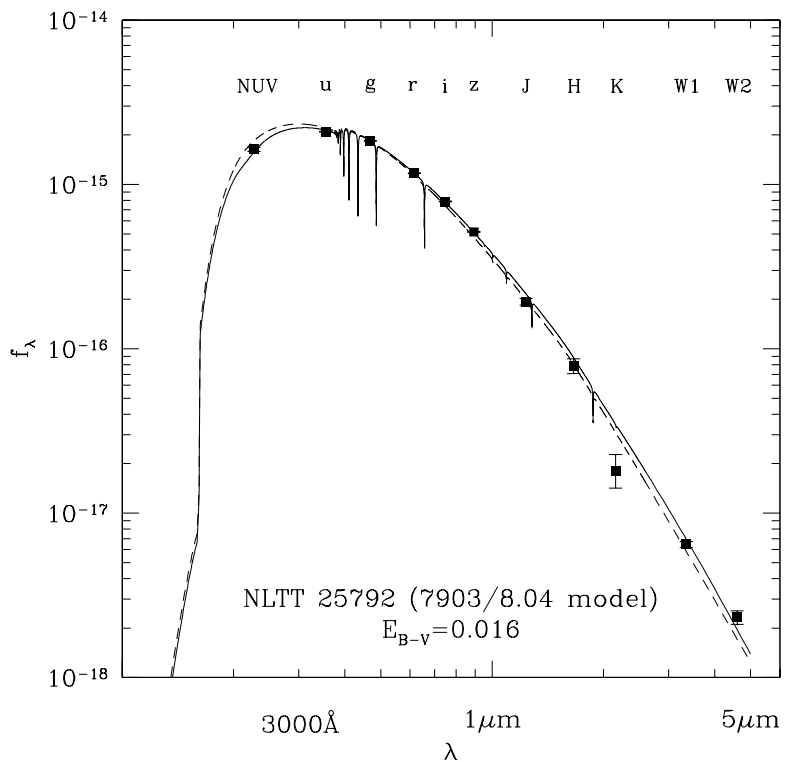

Figure 3. Spectral energy distribution, $f_{\lambda}\left(\mathrm{erg} \mathrm{cm}^{-2} \mathrm{~s}^{-1} \AA^{-1}\right)$ vs $\lambda$, of the DAZ NLTT 25792 from the near UV to the nearIR (Table 1). The data are compared to the best-fit model to the Balmer lines including the effect of interstellar extinction (full line) or excluding it (dashed line). The K-band shows a possible flux deficit. The GALEX NUV and SDSS $u$ magnitudes are best fit with the model including interstellar extinction.

strain the CNO abundance because of a lack of practical abundance diagnostics at a temperature of $\sim 7900 \mathrm{~K}$ for NLTT 25792. For example, the O I $\lambda 7773$ triplet remains extremely weak under these conditions.

The systematic effects of effective temperature and surface gravity variations have been investigated. The abundance shifts corresponding to surface gravity shifts of \pm 0.1 dex for a reference model at $T_{\text {eff }}=7900 \mathrm{~K}$ and $\log g=8.0$ are negligible when measuring calcium or aluminum abundances, but amount to $\mp 0.01-0.02$ in the logarithm of the magnesium abundance and $\pm 0.02-0.04$ for iron. The effect of temperature shifts of $\pm 100 \mathrm{~K}$ for the same reference model are \pm 0.06 for calcium and aluminum, and \pm 0.04 for magnesium and iron. In summary, the temperature uncertainty dominates the errors in abundances relative to hydrogen, but because the relevant elements follow the same trends it would not affect abundance ratios.

\subsection{Comparative Analysis}

The abundance analysis of the four related stars is performed for given atmospheric parameters (Table 3). We collected published effective temperature and surface gravity measurements based on Balmer line profile analyses or joint line profile and parallax analyses.

For G74-7 we averaged the temperature and gravity measurements of Billeres et al. (1997), Gianninas et al. (2004), Gianninas et al. (2005), Holberg et al. (2008), Gianninas et al. (2011), and Giammichele et al. (2012) that are based on a Balmer line profile analysis (method 1 ), and compared the results to the average of the measurements of Bergeron et al. (1997), Leggett et al. (1998), and Bergeron et al. (2001) that are based on the parallax of van Altena et al. (1995), optical/IR SEDs, and high-dispersion $\mathrm{H} \alpha$ spectroscopy (method 2). Some of these measurements may well be redundant, but, in general, they should reflect on differing data sources or model atmosphere generations. In this case, the two methods delivered consistent results: $\left(T_{\text {eff }}, \log g\right)=$ $7305 \pm 22,8.07 \pm 0.03)$ with method 1 and $\left(T_{\text {eff }}, \log g\right)=$ $7316 \pm 103,8.02 \pm 0.05)$ with method 2 . Therefore, we adopted the weighted average of all temperature and surface gravity measurements (Table 3). The corresponding abundance measurements differ slightly from pub- 
Table 2

Equivalent widths and line velocities

\begin{tabular}{|c|c|c|}
\hline $\operatorname{Ion}, \lambda(\AA)^{\mathrm{a}}$ & 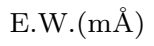 & $v\left(\mathrm{~km} \mathrm{~s}^{-1}\right)^{\mathrm{b}}$ \\
\hline Fe I 3440.606 & 110. & 33.9 \\
\hline Fe I 3565.379 & 37. & 24.1 \\
\hline Fe I 3570.097 & 99. & 25.6 \\
\hline Fe I 3581.193 & 97. & 25.2 \\
\hline Fe I 3719.935 & 105. & 24.7 \\
\hline Fe I 3722.563 & 23. & 22.5 \\
\hline Fe I 3733.317 & 18. & 28.8 \\
\hline Fe I 3734.864 & 110. & 24.7 \\
\hline Fe I 3737.131 & 107. & 22.8 \\
\hline Fe I 3745.561 & 76. & 30.0 \\
\hline Fe I 3748.262 & 30. & 25.2 \\
\hline Fe I 3749.485 & 74. & 25.3 \\
\hline Fe I 3758.233 & 62. & 23.3 \\
\hline Fe I 3763.789 & 37. & 25.3 \\
\hline Fe I 3767.191 & 33. & 27.6 \\
\hline Fe I 3795.002 & 22. & 26.4 \\
\hline Fe I 3804.791 & 19. & 17.4 \\
\hline Fe I 3815.840 & 24. & 24.9 \\
\hline Fe I 3820.425 & 76. & 23.6 \\
\hline Fe I 3824.444 & 32. & 22.8 \\
\hline Fe I 3825.881 & 47. & 19.8 \\
\hline Fe I 3827.823 & 45. & 23.7 \\
\hline Fe I $3829.452^{\text {c }}$ & 37. & 20.7 \\
\hline Mg I 3832.300 & 79 . & 22.7 \\
\hline Fe I 3834.222 & 44. & 28.2 \\
\hline Mg I 3838.292 & 116. & 24.2 \\
\hline Fe I 3856.371 & 25. & 26.3 \\
\hline Fe I 3859.911 & 70. & 23.5 \\
\hline Ca II 3933.660 & 1215. & 19.6 \\
\hline Al I 3944.006 & 23. & 21.3 \\
\hline Al I 3961.520 & 48. & 27.0 \\
\hline Ca II 3968.470 & $\ldots$ & 22.1 \\
\hline Fe I 4045.812 & 64. & 21.5 \\
\hline Fe I 4063.594 & 26. & 23.4 \\
\hline H I 4101.734 & $\ldots$ & 17.0 \\
\hline Ca I 4226.730 & 96. & 26.0 \\
\hline Fe I 4271.760 & 37. & 22.3 \\
\hline H I 4340.462 & $\ldots$ & 19.0 \\
\hline Fe I 4383.545 & 46. & 23.9 \\
\hline Fe I 4404.750 & 32. & 23.3 \\
\hline Fe I 4415.122 & 9. & 23.5 \\
\hline H I 4861.323 & $\ldots$ & 22.6 \\
\hline Fe I 5167.488 & 22 . & 33.5 \\
\hline Mg I 5172.684 & 40. & 27.0 \\
\hline Mg I 5183.604 & 66. & 25.4 \\
\hline H I 6562.797 & $\ldots$ & 26.9 \\
\hline Ca II 8498.020 & 58. & 31.7 \\
\hline Ca II 8542.090 & 273. & 29.5 \\
\hline Ca II 8662.140 & 158. & 29.2 \\
\hline
\end{tabular}

a Laboratory wavelength from the server http://www.nist.gov/pml/data/asd.cfm at the National Institute of Standards and 'Technology (NIST).

b Heliocentric velocities.

c Blended with Mg I $\lambda 3829.3549 \AA$.

lished values: we obtain a lower calcium abundance $(-0.2$ dex) but a higher magnesium abundance (0.3 and 0.1 dex) than in Billeres et al. (1997) and Zuckerman et al. (2003), although our iron and aluminum abundance measurements are in good agreement (within $\approx 0.1 \mathrm{dex}$ ) with those of Zuckerman et al. (2003). The adopted atmospheric parameters in either study are similar to those adopted in this work. Variations may be attributed to different fitting techniques or model generations. The pattern is very nearly scaled on the solar pattern with $[\mathrm{X} / \mathrm{H}] \approx-3.4$, where $\mathrm{X}$ represents $\mathrm{Mg}, \mathrm{Al}, \mathrm{Ca}$, and $\mathrm{Fe}$.

Next, for WD $0354+463$ we used the Balmer line analysis of Gianninas et al. (2011). The effective temperature adopted by Zuckerman et al. (2003) was close to $500 \mathrm{~K}$ cooler and should affect abundance measurements. Predictably, because of the higher temperature adopted in this work our abundance measurements are on average 0.19 dex higher with a dispersion of only 0.08 dex. However, the patterns are similar, except for a slight magnesium enrichment in our data. In addition, and as in the case of G74-7, the calcium abundance measurements based on $\mathrm{Ca} \mathrm{I} \lambda 4226$ and $\mathrm{Ca} \mathrm{K}$ are formally in agreement.

For WD $1257+278$ we averaged the measurements of Gianninas et al. (2011), Limoges \& Bergeron (2010), Holberg et al. (2008), and Liebert et al. (2005) to which we compounded our own analysis of two available SDSS spectra. We excluded a notably defective $\mathrm{H} \alpha$ line from the analysis of one of the SDSS spectrum. We obtained similar parameters (Table 3) to those adopted by Zuckerman et al. (2011). The surface gravity (hence mass) obtained in the joint astrometric/photometric/spectroscopic analysis of Bergeron et al. (2001) is lower than estimated in the cited spectroscopic analyses. Comparing the last two columns of Table 3 we conclude that the effect on the abundance analysis of the surface gravity uncertainty is negligible. However, Zuckerman et al. (2011) list the following abundances for WD $1257+278:[\mathrm{Mg} / \mathrm{H}]=-2.80$, $[\mathrm{Al} / \mathrm{H}]=-2.63,[\mathrm{Ca} / \mathrm{H}]=-2.37$, and $[\mathrm{Fe} / \mathrm{H}]=-2.88$. Their adopted stellar parameters are nearly identical to ours $\left(T_{\text {eff }}=8600 \pm 100 \mathrm{~K}, \log g=8.10 \pm 0.15\right)$. Apart from similar iron abundances, the abundances of $\mathrm{Mg}$, $\mathrm{Al}$, and $\mathrm{Ca}$ are approximately a factor of two lower in our work although we employed the same data set. Such discrepancies may, in part, be caused by differences in the model atmospheres or by different line measurement techniques. The equivalent width measurement of weak lines are notably affected by the choice of the integration window that may inadvertently include neighboring lines or uncalibrated continuum variations. Abundance measurements based on a few lines may suffer from such systematic effects that, however, would tend to average out when including many spectral lines in the abundance measurement. Spectral line fitting may still suffer from poor continuum placement but the line integration is necessarily confined to the width of the synthetic line profile. We noted that the present iron abundance measurement and that of Zuckerman et al. (2011) are based on numerous lines, possibly averaging out systematic effects, and are formally in agreement. Overall, and in agreement with Zuckerman et al. (2011), we found that the atmosphere of WD $1257+278$ appears relatively rich in calcium, particularly relative to magnesium. The calcium abundance measured from the $\mathrm{Ca} \mathrm{K}$ line, $[\mathrm{Ca} / \mathrm{H}]=-2.53 \pm 0.04$, is 0.16 dex higher than measured using the $\mathrm{Ca} \mathrm{I} \lambda 4226$ line and the Ca K line core is poorly fitted, although the overall line profile is well matched. This slight abundance discrepancy may suggest an effect similar to that observed in NLTT 25792 although the $\mathrm{Ca} \mathrm{K}$ line in WD $1257+278$ does not show a notable blue shift or asymmetry.

Finally, we averaged the effective temperature and surface gravity measurements of Gianninas et al. (2011), Holberg et al. (2008), Liebert et al. (2005), and our own measurement of WD $1455+298$ based on SDSS spectroscopy. These measurements based on Balmer line profiles are only marginally consistent with the parallax that implies a larger stellar radius hence lower grav- 
Table 3

Properties and abundances

\begin{tabular}{ccccccc}
\hline \hline & NLTT 25792 & a & G74-7 & WD 1455+298 & WD 0354+463 & \multicolumn{2}{c}{ WD 1257+278 a } \\
\hline$T_{\text {eff }}(\mathrm{K})$ & $7903 \pm 16$ & $7306 \pm 22$ & $7383 \pm 19$ & $8240 \pm 120$ & $8609 \pm 20$ & $(8600)$ \\
$\log g_{\left(\mathrm{cm} \mathrm{s}^{-2}\right)}$ & $8.04 \pm 0.03$ & $8.06 \pm 0.02$ & $7.97 \pm 0.03$ & $7.96 \pm 0.10$ & $8.24 \pm 0.02$ & $(8.0)$ \\
$\operatorname{log~Mg/H~}$ & $-7.24 \pm 0.05$ & $-7.79 \pm 0.06$ & $-8.03 \pm 0.06$ & $-6.70 \pm 0.05$ & $-7.49 \pm 0.08$ & $-7.51 \pm 0.09$ \\
{$[\mathrm{Mg} / \mathrm{H}]^{\mathrm{b}}$} & $-2.80 \pm 0.05$ & $-3.35 \pm 0.06$ & $-3.59 \pm 0.06$ & $-2.26 \pm 0.05$ & $-3.05 \pm 0.08$ & $-3.07 \pm 0.09$ \\
$\log \mathrm{Al} / \mathrm{H}$ & $-8.16 \pm 0.11$ & $-8.90 \pm 0.20$ & $\ldots$ & $-7.98 \pm 0.13$ & $-8.50 \pm 0.25$ & $-8.50 \pm 0.25$ \\
{$[\mathrm{Al} / \mathrm{H}]^{\mathrm{b}}$} & $-2.62 \pm 0.11$ & $-3.36 \pm 0.20$ & $\ldots$ & $-2.44 \pm 0.13$ & $-2.96 \pm 0.25$ & $-2.96 \pm 0.25$ \\
$\log \mathrm{Ca} / \mathrm{H}$ & $-8.07 \pm 0.06$ & $-9.05 \pm 0.04$ & $-9.51 \pm 0.03$ & $-8.20 \pm 0.03$ & $-8.38 \pm 0.06$ & $-8.39 \pm 0.06$ \\
{$[\mathrm{Ca} / \mathrm{H}]^{\mathrm{b}}$} & $-2.40 \pm 0.06$ & $-3.38 \pm 0.04$ & $-3.84 \pm 0.03$ & $-2.53 \pm 0.03$ & $-2.71 \pm 0.06$ & $-2.72 \pm 0.06$ \\
$\log \mathrm{Fe} / \mathrm{H}$ & $-7.16 \pm 0.04$ & $-8.03 \pm 0.09$ & $-8.40 \pm 0.08$ & $-7.13 \pm 0.11$ & $-7.47 \pm 0.09$ & $-7.45 \pm 0.10$ \\
{$[\mathrm{Fe} / \mathrm{H}]^{\mathrm{b}}$} & $-2.63 \pm 0.04$ & $-3.50 \pm 0.09$ & $-3.87 \pm 0.08$ & $-2.60 \pm 0.11$ & $-2.94 \pm 0.09$ & $-2.92 \pm 0.10$ \\
\hline
\end{tabular}

a The calcium abundance measurement excludes $\mathrm{Ca} H \& \mathrm{~K}$.

b $[\mathrm{X} / \mathrm{H}]=\log \mathrm{X} / \mathrm{H}-\log \mathrm{X} / \mathrm{H}_{\odot}$.

ity: WD $1455+298$ is a suspected double degenerate (Bergeron et al. 2001). However, $\mathrm{H} \beta$ and other lines only show single components. Moreover, the SED (Appendix 1) does not show evidence of a cool companion. This discrepancy remains unresolved. The atmosphere of WD $1455+298$ is the cleanest of the sample with an average metallicity index of only $[\mathrm{X} / \mathrm{H}]=-3.7$ where $\mathrm{X}$ represents $\mathrm{Mg}$, Ca, and Fe. Our new abundance measurements differ on average with those of Zuckerman et al. (2003) by only -0.1 dex but with a dispersion of 0.2 dex. The increased signal to noise ratio in recent KOA data resulted in more accurate abundance measurements and a clear detection of the Ca I $\lambda 4226$ line. The present analysis indicates a modest enrichment in magnesium relative to calcium and iron. The present calcium and iron abundance measurements in WD $1455+298$ supercede those presented earlier in Kawka et al. (2011).

Figure 4 shows the abundance patterns in the five objects analyzed. The patterns for both G74-7, WD $0354+463$, and WD $1455+298$ do not suggest calcium enhancement, but those of NLTT 25792 and WD $1257+278$ show a clear enhancement relative to all other elements. The calcium to magnesium ratio is the most revealing with an enhancement relative to solar of +0.40 in NLTT 25792 and +0.34 in WD $1257+278$. Interestingly, the patterns for WD $0354+463$ and WD $1455+298$ show a reversed trend with calcium at its lowest abundance relative to magnesium in the sample, $\approx-0.27$ and -0.25 below solar. Overall, the abundance pattern in G74-7 is flat, with a slightly lower iron abundance than the average pattern. Diffusion effects, i.e., the effects of varying diffusion time scales on the observed abundances, are likely to alter the observed abundance pattern relative to the supplied, i.e, accreted pattern (see a discussion in Section 4).

\subsection{A Ca II Nebular Component?}

Modelling of the Ca K line remains unsatisfactory: The line profile appears deeper and blue-shifted relative to the best fit model to all calcium lines (Fig. 5). Incidentally, the line did not vary in strength between the FORS1 and $\mathrm{X}$-shooter observations.

The line width is dominated by collisions with hydrogen atoms. At the temperature and density conditions

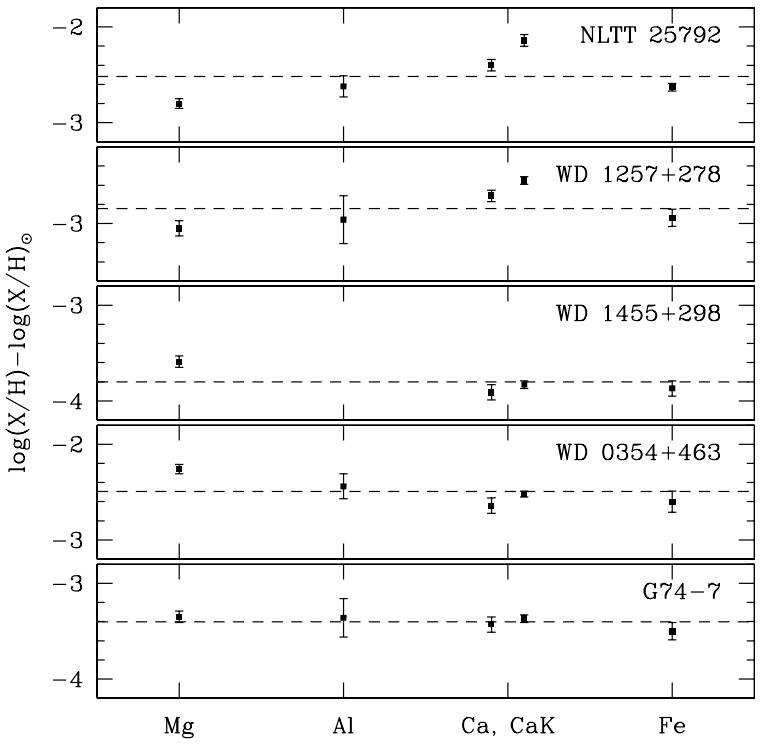

Figure 4. Comparative analysis of magnesium, aluminum, calcium, and iron abundances in the five selected stars. The Ca abundance refers to abundance indicators other than Ca H\&K, i.e, Ca I $\lambda 4226$ or the Ca II IR triplet. The pattern observed in NLTT 25792 is similar to that of WD $1257+278$ and notably dissimilar to the other patterns (see Section 3.4).

prevalent in the atmosphere of NLTT 25792, we estimate that $80 \%$ of the total width $\Gamma_{\text {tot }}$ is contributed by hydrogen atoms and only $20 \%$ by electrons. Increasing $\Gamma_{\text {tot }}$ by a factor of two does increase the equivalent width by $33 \%$ although it does not displace the line further toward the blue. On the other hand, strong lines, such as the Balmer or the Ca H\&K lines, are visibly affected by the atmospheric structure over a wide range of depths, but we noted that the $\mathrm{H} \alpha$ and $\mathrm{H} \beta$ line wings and deep cores measured with X-shooter are well modeled. An accumulation of calcium above the convection zone could result in a stronger line core than predicted by our homogeneous models.

Alternatively, the excess absorption in the Ca K line and the velocity offset could be interpreted as evidence of a nebular component to the observed profile with an 
equivalent width of $\approx 480 \mathrm{~m} \AA$ and at a relative velocity of $-20 \mathrm{~km} \mathrm{~s}^{-1}$. Correcting for the gravitational redshift $\left(\sim 30 \mathrm{~km} \mathrm{~s}^{-1}\right)$, the average velocity of the gas relative to the surface is $+10 \mathrm{~km} \mathrm{~s}^{-1}$. Adopting velocity dispersion appropriate for local interstellar gas at $T \sim 7000 \mathrm{~K}$, i.e., $\sigma=1.7 \mathrm{~km} \mathrm{~s}^{-1}$, an exceedingly large column density $\log N\left(\mathrm{Ca} \mathrm{II} \mathrm{cm}^{-2}\right) \gtrsim 15$ would be required to fill in the observed absorption at a relative velocity of $-20 \mathrm{~km} \mathrm{~s}^{-1}$. However, allowing a larger velocity dispersion of $\sigma=30 \mathrm{~km} \mathrm{~s}^{-1}$, i.e., typical of the range of projected orbital velocity for gas transiting in front of the stellar disk (see Debes et al. 2012), a lower density of $\log N\left(\mathrm{Ca} \mathrm{II} \mathrm{cm}^{-2}\right) \approx 12.8$ is found. This simple geometrical effect helps locate the gas at a radius $r=\left(R_{w d} / \sigma\right)^{2 / 3}\left(G M_{w d}\right)^{1 / 3} \approx 20 R_{w d}$ well within a tidal disruption radius of $100 R_{w d}$. Note that in this analysis it would be more appropriate to adopt rotational broadening function rather than Gaussian velocity distribution.

An origin in the interstellar medium is unlikely. The absorption largely exceeds measurements of interstellar Ca II K line widths $(<300 \mathrm{~m} \AA)$ up to distances of 400 pc (Welsh et al. 2010). Assuming a maximum Ca II volume density of $10^{-8} \mathrm{~cm}^{-3}$, the total column density at the distance of NLTT $25792(d \approx 36 \mathrm{pc})$ would be $10^{12}$ ions $\mathrm{cm}^{-2}$. In conditions typical of the local ISM, the corresponding line equivalent width would not exceed $50 \mathrm{~m} \AA$. At a lower, typical volume density of $10^{-9} \mathrm{~cm}^{-3}$ the column density is $10^{11} \mathrm{~cm}^{-2}$ and the corresponding equivalent width is only a few $m \AA$. It is therefore unlikely that the excess Ca II absorption would originate in the interstellar medium. We conclude that it probably originates in a gaseous circumstellar environment. The presence of ionized gas in the circumstellar environment of DAZ white dwarfs is well documented (see, e.g., Gänsicke et al. 2006; Melis et al. 2012; Debes et al. 2012) and revealed mostly by IR calcium triplet emission, although Gänsicke et al. (2012) noted excess Si IV absorption in ultraviolet spectra of the hot DAZ PG $0843+516$ that could also originate in a hot circumstellar environment. The X-shooter spectra of NLTT 25792 show the IR calcium triplet in absorption. The DAZ WD $1257+278$ also shows excess absorption in the Ca H\&K lines although the HIRES spectrum does not show an obvious line shift or asymmetry. Again, the absorption profile resulting from transiting gas follows a rotational broadening function that may be hidden within the combined profile.

The presence of circumstellar Ca K line in DAZ spectra is analogous to C IV absorption in the circumstellar environment of hot white dwarfs. Ionized species of carbon and silicon are found in circumstellar environment of hot white dwarfs. Bannister et al. (2003) ascribed those features to a Strömgren sphere in the interstellar medium excited by ultraviolet radiation emanated by the white dwarf, although Dickinson et al. (2012) also cite the possibility of evaporating circumstellar debris in the intense ultraviolet radiation field. Clearly, young white dwarfs may be surrounded by even denser material than their older, DAZ counterparts.

\section{SUMMARY AND DISCUSSION}

We measured the metallicity in the atmosphere of the DAZ white dwarf NLTT 25792 based on the detec-

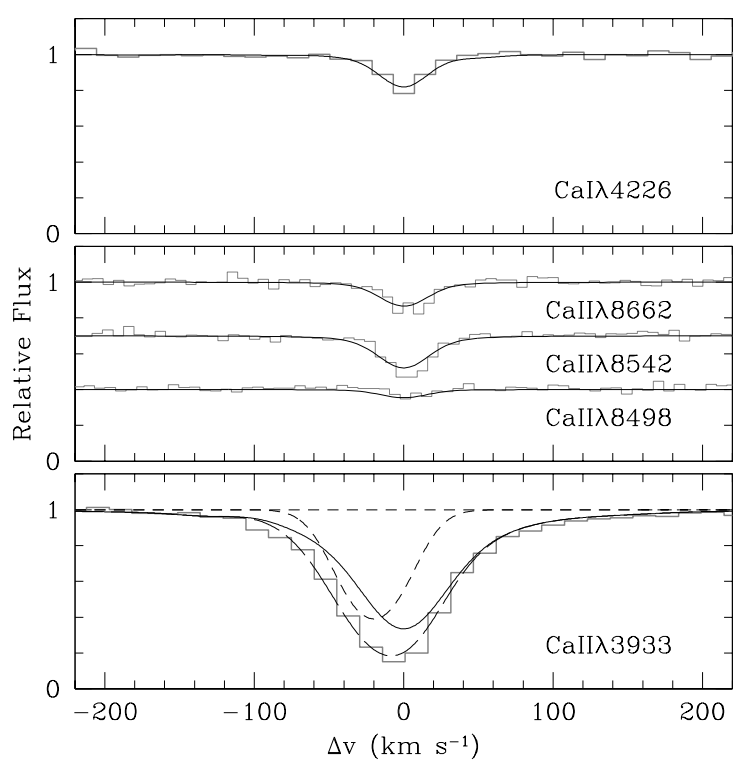

Figure 5. The calcium lines in the $\mathrm{X}$-shooter spectra (grey lines) are compared to spectral line syntheses (full lines) with $[\mathrm{Ca} / \mathrm{H}]=$ -2.4 (Table 3). The $\mathrm{Ca} \mathrm{K}$ line is stronger than predicted by the model and shows a possible nebular component shifted by -20 $\mathrm{km} \mathrm{s}^{-1}$ (short dashed line) that we added to the total profile (long dashed line).

tion of magnesium, aluminum, calcium and iron lines in X-shooter spectra of this object. The average abundance of these elements relative to solar on a logarithmic scale is $[\mathrm{X} / \mathrm{H}] \approx-2.5 \mathrm{dex}$. On the same scale, the upper limits to the sodium and silicon abundances are $[\mathrm{Na} / \mathrm{H}] \lesssim-3.1$ and $[\mathrm{Si} / \mathrm{H}] \lesssim-3.0$. The atmosphere of NLTT 25792 appears relatively rich in calcium, but relatively poorer in sodium, magnesium, and silicon. Also, the $\mathrm{Ca} \mathrm{K}$ line appeared both deeper and offset than predicted by our models suggesting the presence of circumstellar gas. The absence of IR calcium triplet emission implies a lack of ionizing radiation concordant with the relatively low effective temperature of NLTT 25792 $\left(T_{\mathrm{eff}} \approx 7900 \mathrm{~K}\right)$ compared to other DAZ white dwarfs with gaseous disks ( $T_{\text {eff }} \gtrsim 20,000 \mathrm{~K}$, see, e.g., Gänsicke et al. 2006), or their He-rich counterparts with comparable ionizing flux (e.g., SDSS J0738+1835 with $T_{\text {eff }} \approx 14,000 \mathrm{~K}$, Dufour et al. 2012). Disk modeling by Hartmann et al. (2011) shows that emission lines, such as the IR calcium triplet, occurs within the gaseous disk at temperatures of $T_{\text {disk }} \lesssim 7000 \mathrm{~K}$ and inside the tidal radius.

Chemical diversity in the atmosphere of accreting white dwarfs may be attributed to diversity in source compositions. For example, the DAZ NLTT 43806 (Kawka \& Vennes 2006) appears to be iron deficient prompting Zuckerman et al. (2011) to propose that this deficiency along with a calcium/aluminum enrichment implies that the white dwarf may be accreting predominantly "earth-type lithosphere" material. Moreover, Gänsicke et al. (2012) found evidence of chemical diversity in a sample of warm $(\sim 20000 \mathrm{~K})$ white dwarfs, particularly in the form of an overabundance of iron that implies differentiation in the accretion flow.

Another explanation for chemical diversity is the time dependence of the accretion flow and atmospheric diffu- 
sion. Diffusion time-scales at the bottom of the convection zone (see Koester 2009) 2 range from $\tau \sim 10^{3}$ to $10^{4}$ yrs for a hydrogen-rich white dwarf with an effective temperature close to $8000 \mathrm{~K}$ such as NLTT 25792 . This time-scale is nearly instantaneous relative to the age of the white dwarf $\left(t_{\text {cool }} \approx 10^{9}\right.$ yrs $)$. However, we may well speculate that a time scale of $10^{3}$ yrs is comparable or longer than the time lapse between single accretion events that would result in time-variable abundances.

This complicated history may be summarized with two extreme examples related to calcium and iron: in the first, our observations took place shortly $(t<<\tau)$ after the accretion event and the abundances simply reflect the accretion source. In this case, the body accreted onto NLTT 25792 must have been moderately enriched in calcium relative to iron. In the second example, the observations took place long after the accretion event $(t>\tau)$ and, following Koester (2009), the abundances follow an exponential decline $X / H \propto e^{-t / \tau}$, so that the abundance ratio of element $\mathrm{X}$ relative to $\mathrm{Y}$ follows:

$$
\log \mathrm{X} / \mathrm{Y}-\log \mathrm{X} / \mathrm{Y}_{\text {source }}=-\frac{t}{\ln 10}\left(\frac{1}{\tau_{\mathrm{X}}}-\frac{1}{\tau_{\mathrm{Y}}}\right) .
$$

If $\tau_{\mathrm{X}}>\tau_{\mathrm{Y}}$, as in the case of calcium (X) versus iron $(\mathrm{Y})$, then $\mathrm{X}$ (calcium) would gradually dominate over $\mathrm{Y}$ (iron) on a time-scale comparable to diffusion timescales. Numerically, $\tau_{\mathrm{Ca}} / \tau_{\mathrm{Fe}} \approx 1.3-1.5$ in conditions appropriate for NLTT 25792, so that before all elements disappear from the atmosphere at, say, $t \approx \tau_{\mathrm{Ca}}$ the calcium abundance would be enhanced by a factor of 1.4 to 1.6 relative to initial conditions. Assuming initially solar abundances this calcium-to-iron abundance ratio is nearly the ratio observed in NLTT $25792(\approx 1.5$, see Table (3) and there would be no need to assume a calciumrich accretion source. A similar exercise involving the calcium to magnesium abundance ratio necessarily implies a shortage of magnesium in the accretion source because their respective diffusion time-scales are nearly equal. The same situation holds for sodium.

In steady state accretion, the observed abundance ratios are simply given by

$$
\frac{\mathrm{X}}{\mathrm{Y}}=\frac{\mathrm{X}}{\mathrm{Y}_{\text {source }}} \frac{\tau_{\mathrm{X}}}{\tau_{\mathrm{Y}}}
$$

In this case we would have expected an excess of 0.11 to 0.17 dex of the calcium to iron ratio relative to a source assumed to be solar, i.e., close to excess observed in NLTT $25792(0.23 \pm 0.10)$. However, examining other abundance ratios should help constrain the abundance pattern of the accretion source. For example, sodium, magnesium, and calcium have nearly identical diffusion time scales but we found a significant deficit in sodium and magnesium relative to solar with $[\mathrm{Na} / \mathrm{Ca}] \lesssim-0.7$ and $[\mathrm{Mg} / \mathrm{Ca}]=-0.4$. Diffusion time scales for silicon are uncertain. The calculations of Koester (2009) indicate a longer time scale for silicon than calcium, although the recent on-line data indicate the opposite. In either case, the silicon time scale is longer than that of iron for conditions found in NLTT 25792 implying that the silicon deficit $([\mathrm{Si} / \mathrm{Fe}] \lesssim-0.4$, $[\mathrm{Si} / \mathrm{Ca}] \lesssim-0.6)$ can only be

\footnotetext{
2 See also updated time-scale calculations on-line at http://www1.astrophysik.uni-kiel.de/ koester/astrophysics/ and dated January 2013.
}

explained by its absence in the accretion source. Assuming steady state, we conclude that the accretion source shows a deficit in sodium, magnesium and silicon relative to calcium and iron. The aluminum abundance does not significantly depart from solar abundance ratios. Interestingly, Koester et al. (2011) finds that a deficit in sodium relative to solar in some DZ stars would occur while accreting "bulk Earth" material, although an explanation for a similar deficit in silicon is not forthcoming.

In summary, and following Koester (2009), the estimated steady-state accretion rates onto the white dwarf NLTT 25792 are $\dot{M}_{\mathrm{Mg}}=4.6 \times 10^{7} \mathrm{~g} \mathrm{~s}^{-1}, \dot{M}_{\mathrm{Ca}}=1.2 \times$ $10^{7} \mathrm{~g} \mathrm{~s}^{-1}$, and $\dot{M}_{\mathrm{Fe}}=1.7 \times 10^{8} \mathrm{~g} \mathrm{~s}^{-1}$, and using on-line data from D. Koester we estimate $\dot{M}_{\mathrm{Al}}=5.3 \times 10^{6} \mathrm{~g} \mathrm{~s}^{-1}$.

This limited DAZ sample already suggests that the circumstellar environment varies significantly. Abundance ratios, more particularly $[\mathrm{Mg} / \mathrm{Ca}]$, vary considerably within this sample, from $[\mathrm{Mg} / \mathrm{Ca}]=-0.4$ in NLTT 25792 to $[\mathrm{Mg} / \mathrm{Ca}]=+0.27$ in WD $0354+463$. The reasons for these variations are not known. The ratios $[\mathrm{Na} / \mathrm{Ca}]$ and [Si/Ca] in NLTT 25792 fall well below solar ratios and this deficit must originate in the accretion source, although the precise circumstances surrounding accretion of circumstellar material onto cool white dwarfs remain uncertain. In this context, a correlation found between condensation temperature of accreted constituents and corresponding photospheric abundances in a helium-rich polluted white dwarf (DBZ) may offer come clues to the exact nature of the accretion mechanism (Dufour et al. 2012). In a broader context, the calcium to iron abundance ratio in DZ and DAZ stars alike is known to vary by well over an order of magnitude (see Jura \& Xu 2013) although we find this ratio to be rather homogeneous within our sample. On the other hand, Koester et al. (2011) measured large dispersions ( 0.4 to $0.6 \mathrm{dex})$ in the abundance ratio distributions $(\mathrm{Na}, \mathrm{Mg}, \mathrm{Ca}$, and $\mathrm{Fe})$ of a large DZ sample. The present study shows that such variations occur between magnesium and other elements within a sample of closely related DAZ white dwarfs.

New spectroscopy with a higher dispersion than achieved with $\mathrm{X}$-shooter $(R \approx 9000)$ would be useful in resolving the $\mathrm{Ca} \mathrm{K}$ line profile into its photospheric and circumstellar components, if any. More importantly, the existence of polluted, magnetic white dwarfs (e.g., NLTT 10480 and NLTT43806; Kawka \& Vennes 2011; Zuckerman et al. 2011) may be linked to fieldgenerating accretion or interaction events (Tout et al. 2008; Nordhaus et al. 2011). The detection of magnetic fields weaker than $20 \mathrm{kG}$ is not possible at the resolution achieved with X-shooter but high-dispersion spectroscopy of cool DAZ white dwarfs may help reinforce a link between accretion events and magnetic field generation in compact objects.

A.K. and S.V. acknowledge support from the Grant Agency of the Czech Republic (P209/12/0217 and 13$14581 \mathrm{~S})$. This work was also supported by the project RVO:67985815 in the Czech Republic. We thank the referee for suggesting several improvements to the paper.

This research has made use of the Keck Observatory Archive (KOA), which is operated by the W. M. Keck Observatory and the NASA Exoplanet Science Institute 
(NExScI), under contract with the National Aeronautics and Space Administration.

This publication makes use of data products from the Wide-field Infrared Survey Explorer, which is a joint project of the University of California, Los Angeles, and the Jet Propulsion Laboratory/California Institute of Technology, funded by the National Aeronautics and Space Administration, and from the Two Micron All Sky Survey, which is a joint project of the University of Massachusetts and the Infrared Processing and Analysis Center/California Institute of Technology, funded by the National Aeronautics and Space Administration and the National Science Foundation.

Facilities: VLT:Kueyen

\section{REFERENCES}

Abazajian, K. N., Adelman-McCarthy, J. K., Agüeros, M. A., et al. 2009, ApJS, 182, 543

Asplund, M., Grevesse, N., Sauval, A. J., \& Scott, P. 2009, ARA\&A, 47, 481

Bannister, N. P., Barstow, M. A., Holberg, J. B., \& Bruhweiler, F. C. 2003, MNRAS, 341, 477

Barklem, P. S., Piskunov, N., \& O'Mara, B. J. 2000, A\&AS, 142, 467

Bergeron, P., Leggett, S. K., \& Ruiz, M. T. 2001, ApJS, 133, 413 Bergeron, P., Ruiz, M. T., \& Leggett, S. K. 1997, ApJS, 108, 339 Billeres, M., Wesemael, F., Bergeron, P., \& Beauchamp, A. 1997, ApJ, 488, 368

Cardelli, J. A., Clayton, G. C., \& Mathis, J. S. 1989, ApJ, 345, 245

Cushing, M. C., Rayner, J. T., \& Vacca, W. D. 2005, ApJ, 623, 1115

Cutri, R. M., et al. 2012, VizieR Online Data Catalog, 2311, 0 (wise.rept, $1 \mathrm{C}$ )

Debes, J. H. 2006, ApJ, 652, 636

Debes, J. H., Kilic, M., Faedi, F., et al. 2012, ApJ, 754, 59

Dickinson, N. J., Barstow, M. A., Welsh, B. Y., et al. 2012, MNRAS, 423, 1397

Dufour, P., Bergeron, P., Liebert, J., et al. 2007, ApJ, 663, 1291

Dufour, P., Kilic, M., Fontaine, G., et al. 2012, ApJ, 749, 6

Dupuis, J., Fontaine, G., Pelletier, C., \& Wesemael, F. 1992, ApJS, 82, 505

Farihi, J., Hoard, D. W., \& Wachter, S. 2006, ApJ, 646, 480

Farihi, J., Jura, M., Lee, J.-E., \& Zuckerman, B. 2010, ApJ, 714, 1386

Farihi, J., Jura, M., \& Zuckerman, B. 2009, ApJ, 694, 805

Farihi, J., Zuckerman, B., \& Becklin, E. E. 2008, ApJ, 674, 431

Gänsicke, B. T., Koester, D., Farihi, J., et al. 2012, MNRAS, 424, 333

Gänsicke, B. T., Marsh, T. R., \& Southworth, J. 2007, MNRAS, 380, L35

Gänsicke, B. T., Marsh, T. R., Southworth, J., \&

Rebassa-Mansergas, A. 2006, Science, 314, 1908

Giammichele, N., Bergeron, P., \& Dufour, P. 2012, ApJS, 199, 29

Gianninas, A., Bergeron, P., \& Fontaine, G. 2005, ApJ, 631, 1100

Gianninas, A., Bergeron, P., \& Ruiz, M. T. 2011, ApJ, 743, 138

Gianninas, A., Dufour, P., \& Bergeron, P. 2004, ApJ, 617, L57
Greenstein, J. L., \& Liebert, J. W. 1990, ApJ, 360, 662

Hartmann, S., Nagel, T., Rauch, T., \& Werner, K. 2011, A\&A, 530, A7

Holberg, J. B., Bergeron, P., \& Gianninas, A. 2008, AJ, 135, 1239

Jura, M. 2003, ApJ, 584, L91

Jura, M. 2008, AJ, 135, 1785

Jura, M., \& Xu, S. 2013, AJ, 145, 30

Kawka, A. \& Vennes, S. 2006, A\&A, 643, 402

Kawka, A., \& Vennes, S. 2011, A\&A, 532, A7

Kawka, A., \& Vennes, S. 2012a, MNRAS, 425, 1394

Kawka, A., \& Vennes, S. 2012b, A\&A, 538, A13

Kawka, A., Vennes, S., Dinnbier, F., Cibulková, H., \& Németh, P. 2011, American Institute of Physics Conference Series, 1331, 238

Kawka, A., Vennes, S., Dupuis, J., Chayer, P., \& Lanz, T. 2008, ApJ, 675, 1518

Kilic, M., von Hippel, T., Leggett, S. K., \& Winget, D. E. 2006, ApJ, 646, 474

Kilkenny, D., O’Donoghue, D., Koen, C., Stobie, R. S., \& Chen, A. 1997, MNRAS, 287, 867

Kirkpatrick, J. D., \& McCarthy, D. W., Jr. 1994, AJ, 107, 333

Koester, D. 2009, A\&A, 498, 517

Koester, D., Girven, J., Gänsicke, B. T., \& Dufour, P. 2011, A\&A, 530, A114

Koester, D., Rollenhagen, K., Napiwotzki, R., et al. 2005, A\&A, 432,1025

Lacombe, P., Wesemael, F., Fontaine, G., \& Liebert, J. 1983, ApJ, 272, 660

Leggett, S. K., Ruiz, M. T., \& Bergeron, P. 1998, ApJ, 497, 294

Liebert, J., Bergeron, P., \& Holberg, J. B. 2005, ApJS, 156, 47

Limoges, M.-M., \& Bergeron, P. 2010, ApJ, 714, 1037

Lodders, K., Palme, H., \& Gail, H.-P. 2009, Landolt-Börnstein, New Series, VI/4B, 560

Melis, C., Dufour, P., Farihi, J., et al. 2012, ApJ, 751, L4

Nordhaus, J., Wellons, S., Spiegel, D. S., Metzger, B. D., \&

Blackman, E. G. 2011, Proceedings of the National Academy of Science, 108, 3135

Rayner, J. T., Cushing, M. C., \& Vacca, W. D. 2009, ApJS, 185, 289

Salim, S., \& Gould, A. 2003, ApJ, 582, 1011

Schlegel, D. J., Finkbeiner, D. P., \& Davis, M. 1998, ApJ, 500, 525

Skrutskie, M. F., Cutri, R. M., Stiening, R., et al. 2006, AJ, 131, 1163

Steele, P. R., Saglia, R. P., Burleigh, M. R., et al. 2013, MNRAS, 429, 3492

Thorstensen, J. R. 2003, AJ, 126, 3017

Tinney, C. G., \& Reid, I. N. 1998, MNRAS, 301, 1031

Tout, C. A., Wickramasinghe, D. T., Liebert, J., Ferrario, L., \& Pringle, J. E. 2008, MNRAS, 387, 897

van Altena, W. F., Lee, J. T., Hoffleit, E. D. 1995, The general catalogue of trigonometric parallaxes, New Haven, CT: Yale University Observatory, 4th ed.

Vernet, J., et al. 2011, A\&A, 536, A105

Welsh, B. Y., Lallement, R., Vergely, J.-L., \& Raimond, S. 2010, A\&A, 510, A54

Zuckerman, B., Koester, D., Dufour, P., et al. 2011, ApJ, 739, 101

Zuckerman, B., Koester, D., Reid, I. N., Hünsch, M. 2003, ApJ, 596,477

Zuckerman, B., Melis, C., Klein, B., Koester, D., \& Jura, M. 2010, ApJ, 722, 725

\section{APPENDIX}

The SEDs of the four related stars (Fig. 6) reveal two apparently single white dwarfs without clear evidence of circumstellar environments and one single white dwarf with a faint dusty environment (WD 1455+298; Farihi et al. 2008), while the SED of WD 0354+463 shows a dM7 companion. The SEDs were built using GALEX NUV, SDSS ugriz, 2MASS JHK, and WISE photometric measurements (see Section 2). Optical data for WD $0354+463$ are from Greenstein \& Liebert (1990) using the multichannel spectrophotometer (MCSP), and from Holberg et al. (2008) for WD $0208+396$.

The data are compared to predicted SED based on adopted stellar parameters (Table 3). 

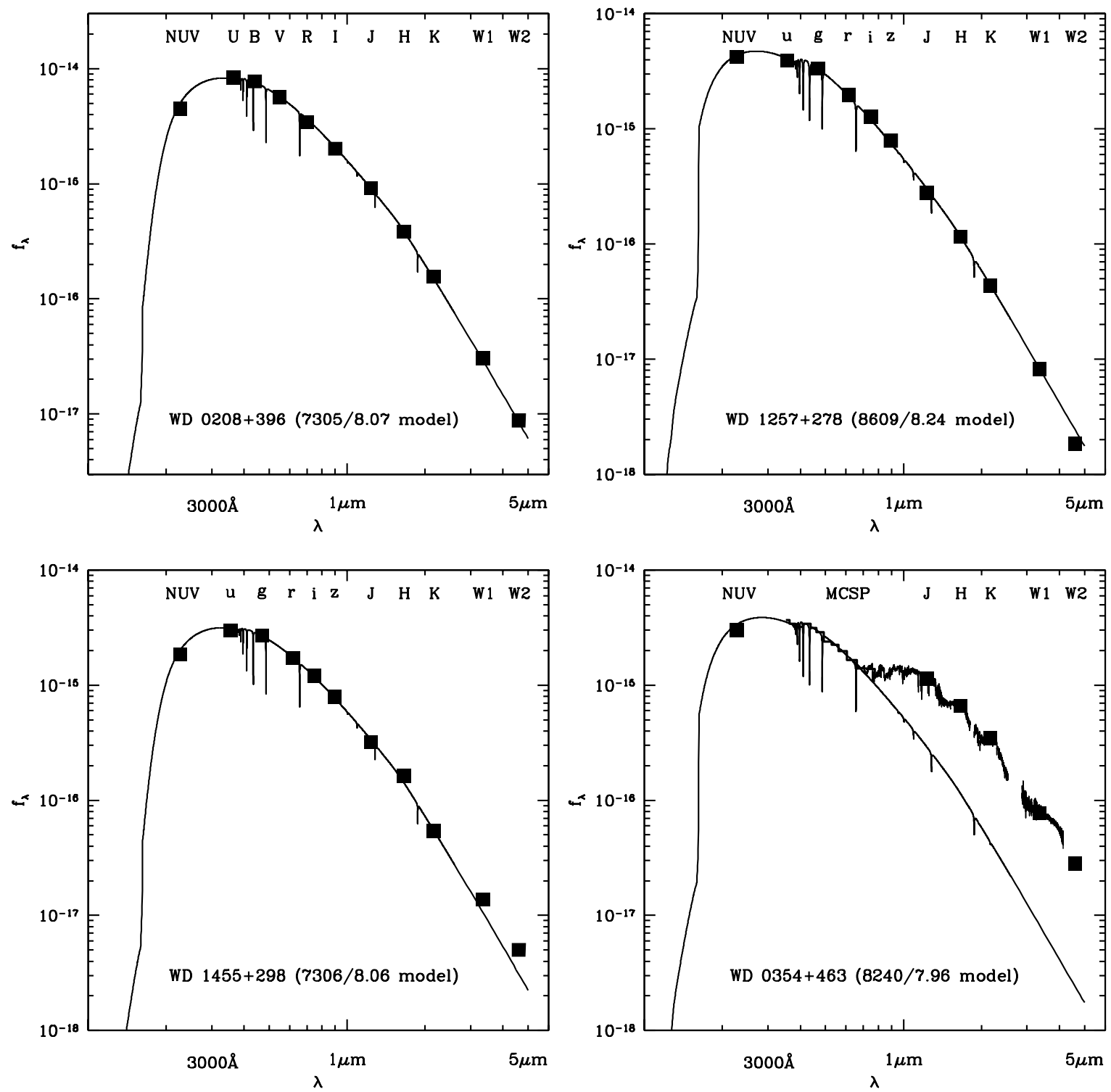

Figure 6. Spectral energy distributions of the four related stars. The excess in WD $0354+463$ is due to the dM7 companion, while a weak near-IR excess is apparent in the WISE observations of WD 1455+298. 